\title{
Disfunções urinárias em mulheres praticantes de atividade física em academias - um estudo transversal
}

\author{
Urinary dysfunction in women practicing physical activity in \\ academies - a cross-sectional study
}

\author{
Leidiany Bueno da Silva', Wyrla Oliveira Santos ${ }^{2}$, Nazete dos Santos Araujo ${ }^{3}$, \\ Cibele Nazaré Camara Rodrigues ${ }^{4}$, Erica Feio Carneiro Nunes ${ }^{5}$ \\ 'Universidade da Amazônia. Belém, Pará, Brasil. ORCID: 0000-0002-0220-6729. leidianysilva23@gmail.com \\ ${ }^{2}$ Universidade da Amazônia. Belém, Pará, Brasil. ORCID: 0000-0002-4885-3577. wyrla.oli@gmail.com \\ 3Universidade da Amazônia. Belém, Pará, Brasil. ORCID: 0000-0003-3644-7165. nazetearaujo@hotmail.com \\ ${ }^{4}$ Universidade Federal do Pará. Belém, Pará, Brasil. ORCID: 0000- 0002-2429-9970. cibelecamara@hotmail.com \\ ${ }^{5}$ Autora para correspondência. Universidade do Estado do Pará. Belém, Pará, Brasil. ORCID: 0000-0002-1274-4686. erica@perineo.net
}

RESUMO I INTRODUÇÃO: Segundo a International Continence Society (ICS), a Incontinência Urinaria de Esforço (IUE) é a mais comum causa de perda involuntária de urina que tem como fator de risco, a prática de atividade física e esportiva em mulheres. OBJETIVO: Verificar a prevalência de IUE em mulheres com prática regular de atividade física em academias. METODOLOGIA: Estudo transversal, realizado em duas academias particulares da cidade de Belém do Pará, em mulheres praticantes de atividade física regular, com idade entre 25 e 55 anos; foram excluídas mulheres com alteração cognitiva, portadoras de diabetes e com problemas neurológicos. Foram aplicados um questionário padrão e o Protection, Amount, Frequency, Adjustment and Body image (PRAFAB). As variáveis foram analisadas pelos testes Exato de Fisher e Mann-Whitney $(p<0,05)$. CAAE 61681416.4.0000.5173. RESULTADOS: A amostra foi composta por 56 mulheres, das quais $7(12 \%)$ relataram perda recente de urina. As incontinentes eram praticantes de musculação (100\%) e a maioria $(71 \%)$ associava esta prática com exercícios aeróbicos. As ocorrências foram relatadas em média de 5 vezes por semana $(85 \%)$ e durante os exercícios (57\%). Não houve diferença estatística na comparação das variáveis entre continentes e incontinentes ( $p>0,05)$. CONCLUSÃO: A prevalência de IU em mulheres praticantes de exercícios aeróbicos e musculação nas academias foi baixa (12\%), com severidade de moderada a grave e sem associação com idade, modalidade, tempo de prática, turno e frequência de exercícios.

PALAVRAS-CHAVE: Incontinência urinária. Atividade física. Assoalho pélvico.

\begin{abstract}
I INTRODUCTION: According to the International Continence Society (ICS), the Urinary Incontinence of Effort (UIE) is the most common cause of involuntary loss of urine, which has as a risk factor the practice of physical and sports activity in women. OBJECTIVE: To verify the prevalence of UIE in women with regular practice of physical activity in academies. METHODOLOGY: A crosssectional study, carried out in two private academies in the city of Belém do Pará, in women practicing regular physical activity, aged between 25 and 55 years; women with cognitive impairment, diabetes mellitus, and neurological problems were excluded. A standard questionnaire and the Protection, Amount, Frequency, Adjustment and Body image (PRAFAB) were applied. The variables were analyzed by Fisher's Exact and Mann-Whitney tests ( $p<0.05$ ). CAAE 61681416.4 .0000 .5173 . RESULTS: The sample consisted of 56 women, of whom $7(12 \%)$ reported recent loss of urine. The incontinent women were bodybuilders (100\%) and the majority $(71 \%)$ associated this practice with aerobic exercises. The occurrences were reported on average 5 times per week $(85 \%)$ and during the exercises $(57 \%)$. There was no statistical difference in the comparison of the variables between continents and incontinent women ( $p>0.05$ ). CONCLUSION: The prevalence of $U I$ in women who performed aerobic exercises and bodybuilding in gymnasiums was low (12\%), with moderate to severe severity and no association with age, modality, practical time, shift and exercise frequency.
\end{abstract}

KEYWORDS: Urinary incontinence. Physical activity. Pelvic floor. 


\section{Introdução}

A International Continence Society (ICS) afirma que a Incontinência Urinária (IU) é qualquer perda involuntária de urina ${ }^{1}$ que consiste em uma patologia que resulta em diversos efeitos sobre as atividades diárias como a interação social e percepção da própria saúde. Especialmente está relacionada ao bem-estar social e mental, abrangendo problemas na vida sexual, constrangimento social, baixa autoestima e depressão. Pode ocorrer abandono das práticas esportivas em decorrência da incontinência urinária ${ }^{2}$.

A IU é um problema crescente que afeta 200 milhões de pessoas de todas as idades, sendo a maioria mulheres. Contudo, sabe-se que um quarto das mulheres entre 15 a 64 anos de idade já apresentou algum episódio de IU, e dessas apenas um quarto procura um serviço médico por causa da perda de urina ${ }^{3}$.

Podemos caracterizar a IU de acordo com o evento que leva à perda de urina, e assim classificada em três tipos: incontinência urinária de esforço (IUE), que é considerada uma perda urinária mediante a um esforço, exercício físico, tosse ou espirro; incontinência urinária de urgência (IUU) que leva a perda involuntária de urina acompanhada por súbita e uma incontrolável vontade de urinar, difícil de ser adiada e IU mista na qual ocorrem sinais e sintomas dos dois tipos já citados ${ }^{4}$.

Nos dias atuais, a prática de exercícios físicos nas academias vem se tornando um hábito cada vez mais frequente entre as mulheres ${ }^{5}$. Sabe-se que a prática regular de exercícios físicos aeróbicos, de fortalecimento muscular e de flexibilidade promove benefícios na prevenção e tratamento de várias patologias, como doenças cardíacas, hipertensão arterial sistêmica, obesidade, osteoporose, dentre outras. Os exercícios também influenciam nos aspectos emocionais, propiciando bem-estar psicológico e reduzindo sintomas como ansiedade, estresse e depressão ${ }^{6}$.

Ainda que comprovados os benefícios na qualidade de vida das mulheres, a prática excessiva de atividades físicas pode ser um fator de risco para o desenvolvimento de $\mathrm{IU}^{7,8}$. O tipo mais comum de incontinência em mulheres com idade de 20 a 40 anos é a IUE, pois, exercícios de grande impacto e que demandam intenso esforço, aumentam a pressão intra-abdominal, podendo sobrecarregar os órgãos pélvicos, gerando assim graves danos à musculatura do assoalho pélvico ${ }^{3}$.

Com base no assunto abordado, nota-se a falta de informação acerca da importância da contração da musculatura perineal durante os exercícios físicos, visto que a prevalência da IU em atletas de elite pode chegar a $80 \% 9$ e em mulheres com idade média de 15 a 64 anos que praticam atividades mais simples, pode variar de 10 a $55 \%^{10}$. Assim, podem surgir várias disfunções urinárias e sexuais, decorrentes da inadequada ou não contração desta musculatura.

Considerando que, embora já existam estudos que apontem para $\circ$ risco de IU em praticantes de atividade física, principalmente em desportistas, ainda não está claro se as mulheres que praticam exercícios em academias apresentam risco de desenvolver IU. Desta forma, objetivo deste estudo foi de verificar a prevalência de IU em mulheres praticantes de atividade físicas nas academias de Belém do Pará.

\section{Metodologia}

Trata-se de um estudo transversal realizado nas academias Trainer e Evolution localizadas na cidade de Belém do Pará, durante os meses de março e abril de 2017. O público alvo foi mulheres praticantes de atividade física em academia, que satisfizessem os critérios de elegibilidade. Foram incluídas as que tivessem entre 25 a 55 anos e fossem residentes de Belém. Foram excluídas mulheres que apresentavam alteração cognitiva, portadoras de diabetes e com problemas neurológicos. O trabalho foi aprovado pelo Comitê de Ética em Pesquisa com Seres Humanas da Universidade da Amazônia (parecer 115358/2016; CAAE 61681416.4.0000.5173).

A seleção das voluntárias foi por amostragem por conveniência, sendo as mesmas abordadas individualmente durante a atividade física e convidadas a participar do estudo. Após o aceite, eram submetidas a uma avaliação composta de: 
anamnese, na qual foram colhidos os dados pessoais da participante, questões referentes ao tipo, tempo, turno e a frequência de atividade física realizada. Em seguida respondiam questões sobre os sintomas de IU: se existia a perda de urina e em que momento ocorria essa perda, como era sua progressão, se existiam fatores associados e por último se esse problema afetava sua vida sexual.

Caso a resposta fosse positiva para a presença de IU, a voluntária respondia o questionário Protection, Amount,. Frequency, Adjustment, Body image
(PRAFAB), instrumento não validado, traduzido para língua portuguesa, que teve como objetivo quantificar a severidade da IU. Ele possui 5 domínios proteção, frequência, quantidade, adaptações e autoimagem. $O$ escore de cada domínio varia de 1 a 4, sendo o escore total podendo variar de 5 a 20 . A IU é classificada em leve, moderada e severa da seguinte forma: os escores de 4 a 6 significa IU leve, 7 a 10 moderada e 11 a 20 severa $^{11}$.

O primeiro domínio "proteção" tem correspondência com o Pad test (validado), conforme descrito na tabela 1:

Tabela 1. Severidade da IUE, conforme classificação da $1^{a}$ questão PRAFAB

\begin{tabular}{cccc}
\hline SEVERIDADE & ESCORE & PAD TEST $48 \mathrm{HORAS}$ & USO DE PROTETORES \\
\hline Leve & 1 & $<20 \mathrm{~g}$ & Não usa \\
Moderada & 2 & $20-100 \mathrm{~g}$ & Ocasionalmente \\
Grave & 3 & $100-200 \mathrm{~g}$ & $\begin{array}{c}\text { Constante, exceto } \\
\text { descansar/dormir } \\
\text { Muito grave }\end{array}$ \\
\hline
\end{tabular}

Para o cálculo amostral foram consideradas as populações da academia Trainer (A 1) que tem 270 mulheres matriculadas e da academia Evolution (A2), com 200 mulheres matriculadas. Foi utilizada uma calculadora online para definir a amostra necessária, com um intervalo de confiança (IC) de $95 \%$, com a margem de erro de $7 \%$ e prevalência estimada de 25\%. Para estes parâmetros, foi estimada uma amostra de 147 mulheres.

Para melhor apresentação dos resultados, as participantes foram divididas em dois grupos $G 1$ e $G 2$, sendo $G 1$ o grupo de mulheres que afirmaram que sofria algum tipo de disfunção urinária e - G2 foram as demais mulheres que negaram. Para comparar os dois grupos em relação ao tipo de atividades físicas referidas pelas mulheres entrevistadas foram utilizados o teste $G$, teste exato de Fisher e o teste de Mann-Whitney, de acordo com as características das variáveis. Em todo trabalho foi utilizado o nível de significância de $5 \%$. E as análises estatísticas foram realizadas no programa Bioestat 5.3.

\section{Resultados}

Só aceitaram participar do estudo 56 mulheres, das quais apenas $12 \%$ afirmaram a perda de urina (figura 1).

Figura 1. Prevalência de perda de urina do grupo amostral $(n=12)$

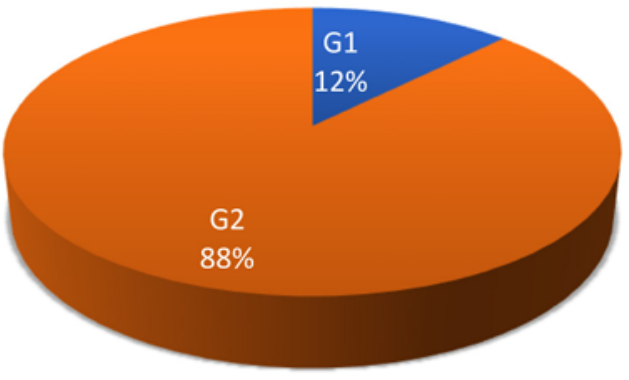


As variáveis idades, o número de atividades que cada participante realiza dentro da academia, se elas realizam atividades físicas fora da academia e nos finais de semana, o tempo de treino e o número de vezes na semana que as mesmas praticam atividade física estão descritas na tabela 2.

Tabela 2: Distribuição das da frequência das variáveis referentes a idade e características das atividades na academia em G1 e G2.

\begin{tabular}{|c|c|c|c|c|c|}
\hline Variáveis & G1 $(n=7)$ & $\%$ & G2 $(n=49)$ & $\%$ & Valor de $p$ \\
\hline \multicolumn{6}{|l|}{ Idade } \\
\hline Média & 45.9 & & 36.3 & & $0.0614^{*}$ \\
\hline Desvio-padrão & 14.3 & & 8.2 & & \\
\hline \multicolumn{6}{|l|}{$N^{\circ}$ de Atividades na academia } \\
\hline 1 & 2 & 28.6 & 20 & 40.8 & $0.3603^{* *}$ \\
\hline 2 & 1 & 14.3 & 15 & 30.6 & \\
\hline$>3$ & 4 & 57.1 & 14 & 28.6 & \\
\hline \multicolumn{6}{|l|}{ Atividade fora da academia } \\
\hline $\operatorname{Sim}$ & 2 & 28.6 & 9 & 18.4 & $0.6116^{* * *}$ \\
\hline Não & 5 & 71.4 & 40 & 81.6 & \\
\hline \multicolumn{6}{|l|}{ Atividade no FDS ${ }^{1}$} \\
\hline Sim & 6 & 85.7 & 41 & 83.7 & $1.000 * * *$ \\
\hline Não & 1 & 14.3 & 8 & 16.3 & \\
\hline \multicolumn{6}{|l|}{ Tempo da atividade } \\
\hline Média & 77.1 & & 29.3 & & $0.6378 *$ \\
\hline Desvio-padrão & 89.8 & & 44.1 & & \\
\hline \multicolumn{6}{|l|}{$N^{\circ}$ de vezes/semana } \\
\hline 1 a 3 vezes & 1 & 14.3 & 10 & 20.4 & $0.5771 * *$ \\
\hline 5 vezes & 6 & 85.7 & 35 & 71.4 & \\
\hline 7 vezes & 0 & 0.0 & 4 & 8.2 & \\
\hline \multicolumn{6}{|l|}{ Turno } \\
\hline Manhã & 2 & 28.6 & 18 & 36.7 & $0.8270 * *$ \\
\hline Tarde & 2 & 28.6 & 16 & 32.7 & \\
\hline Noite & 3 & 42.9 & 15 & 30.6 & \\
\hline
\end{tabular}

'Final de semana

A tabela 3 descreve as atividades realizadas dentro das academias, sendo possível observar que a maioria das participantes praticava musculação, atividade esta que é muito requisitada hoje em dia por várias mulheres que buscam uma melhor qualidade de vida e melhora da autoestima. 
Tabela 3. Atividades exercidas pelas praticantes nas academias

\begin{tabular}{c|c|c|c|c}
\hline Atividade & G1 & $\%$ & G2 & $\%$ \\
\hline Musculação & 7 & 100.0 & 48 & 98.0 \\
Aeróbicos & 5 & 71.4 & 26 & 53.1 \\
Ritmos & 3 & 42.9 & 10 & 20.4 \\
Jump & 2 & 28.6 & 6 & 12.2 \\
Kango Jump & 0 & 0.0 & 1 & 2.0 \\
Pilates & 0 & 0.0 & 4 & 8.2 \\
\hline
\end{tabular}

A tabela 4 descreve o questionário avalição fisioterápica da função urinária que foi entregue apenas para as mulheres que afirmaram a perda de urina. Das 7 mulheres a situação da perda 2 foram exercitando, 2 tossindo, 1 espirrando e 2 de outra maneira não presente no questionário. Todas sentiam quando perdiam e com frequência de uma vez ao dia, sendo que a hora da perda eram 4 pela manhã, 2 pela noite e 1 pela tarde.

Tabela 4. Características da perda urinaria do $G 1(n=7)$.

\begin{tabular}{c|ccccc}
\hline $\begin{array}{c}\text { Situação de } \\
\text { perda }\end{array}$ & $\begin{array}{c}\text { Prevalência } \\
\text { da perda }\end{array}$ & $\begin{array}{c}\text { Quantidade } \\
\text { perdida }\end{array}$ & $\begin{array}{c}\text { Sente ao } \\
\text { perder }\end{array}$ & $\begin{array}{c}\text { Frequência da } \\
\text { perda }\end{array}$ & $\begin{array}{c}\text { Hora da } \\
\text { perda }\end{array}$ \\
\hline Exercitando & 2 & Gotas & Sim & 1 vez ao dia & Noite \\
Outra & 2 & Gotas & Sim & 1 vez ao dia & Manhã \\
Tossindo & 1 & Gotas & Sim & 1 vez ao dia & Manhã \\
Espirrando & 1 & Gotas & Sim & 1 vez ao dia & Manhã \\
Tossindo & 1 & Gotas & Sim & 1 vez ao dia & Tarde \\
\hline
\end{tabular}

A tabela 5 descreve os escores obtidos no questionário PRAFAB que foi respondido pelas participantes com perda urinária. $O$ domínio proteção 5 mulheres responderam que nunca usam protetores (fralda/absorvente) contra a IU e duas falaram que as vezes usam protetores, ou precisam trocar a roupa íntima por conta da IU. No domínio quantidade de perda todas responderam que perdem algumas gotas de urina ou menos, em frequência 6 afirmaram que essa perda ocorre uma vez por semana ou menos e uma disse que é entre uma e três vezes na semana. Todas as participantes afirmaram que isso não prejudica suas atividades diárias, no domínio autoimagem corporal 4 responderam que a IU não lhes incomoda e 3 já disseram que a IU é irritante e problemática, porém não se sentem tão incomodadas por ela.

Tabela 5. Frequência dos scores obtidos em cada variável do PRAFAB (G1=7).

\begin{tabular}{ccc}
\hline Domínio & Número de voluntárias & Score \\
\hline Proteção & 5 & 1 \\
Quantidade da perda de urina & 2 & 2 \\
Frequência da perda de urina & 7 & 1 \\
Adaptações & 6 & 1 \\
& 1 & 2 \\
Imagem corporal & 7 & 1 \\
& 4 & 1 \\
& 3 & 2 \\
Score total & 2 & 5 \\
& 4 & 6 \\
\end{tabular}




\section{Discussão}

Este estudo teve por objetivo verificar a prevalência de IU em mulheres que praticavam atividade física em academias na cidade de Belém do Pará. Nele, foi possivel notar que a prevalência de IU foi baixa, atingindo apenas $12 \%$ das praticantes. No entanto, a atividade física tem sido apontada como um fator risco para adquirir algum tipo de disfunção urinária. ${ }^{6}$ Acredita-se que isto ocorra devido ao comprometimento dos mecanismos de sustentação, suspensão e contenção dos músculos do assoalho pélvico (MAP), que sofrem uma sobrecarga repetida, promovendo assim o enfraquecimento dos mesmos ${ }^{12}$. Alguns exercícios aumentam a pressão intra-abdominal, provocando uma sobrecarga para os MAP, favorecendo o surgimento da perda involuntária de urina ${ }^{13}$.

A maioria das voluntárias, tanto as continentes como as incontinentes, praticava exercício 5 vezes na semana, incluindo os finais de semana. No grupo das incontinentes, a maioria praticava mais de 3 modalidades físicas (musculação, exercícios aeróbicos, Jump e ritmos). Essas modalidades geram alto impacto nos MAP, o que pode ter contribuído para a perda de urina. Pode-se ainda citar a ginástica, atletismo, fisiculturismo, saltos, exercícios que exijam contrações abdominais máximas repetitivas, além de esportes com mudança abrupta de movimento 9,14 como atividades que levam a perda de urina, pois tais atividades resultam em força de impacto sobre o assoalho pélvico três a quatro vezes maior que o peso corporal ${ }^{15}$.

A despeito de não ter sido verificada diferença estatística entre os grupos, acredita-se que atividades físicas intensas estão na gênese da IUE. Os exercícios físicos de alto impacto podem ocasionar em aumento excessivo na pressão intraabdominal, podendo sobrecarregar os órgãos pélvicos, empurrando-os para baixo, ocasionando danos aos músculos responsáveis pelo suporte desses órgãos6. A prevalência dos exercícios de alto impacto pode variar de $28 \%$ a $80 \%{ }^{16}$.

Neste estudo, a média de idade foi maior no grupo que referia perda de urina em relação ao grupo de continentes. A probabilidade das mulheres com idade mais avançada em apresentar disfunções urinárias é maior em relação às de menor idade ${ }^{17}$, devido ao envelhecimento natural das fibras musculares, a redução da função ovariana, o aumento de massa corporal, a gravidez e os múltiplos partos vaginais ${ }^{18}$, com uma prevalência de $30 \%-40 \%$ na meia idade e de $30 \%-50 \%$ nas mulheres mais velhas ${ }^{19}$. Este pode ter sido um fator de confundimento que, devido ao tamanho amostral, não foi possível rodar uma análise multivariada.

O questionário PRAFAB identificou de leve a moderada a severidade da IU, conforme os escores apresentados no domínio proteção. A gravidade da IU já foi avaliada em outro estudo em 59 mulheres praticantes de esportes ${ }^{20}$, das quais 35 eram atletas amadoras de futebol, utilizando o pad test de uma hora e o International Consultation on Incontinence Questionnaire (ICIQ$\mathrm{SF}$ ). Aqueles autores observaram que o grupo de atletas apresentou maior severidade, sem diferença estatística significante entre os grupos pelo Pad test. A maior frequência de sintomas ocorreu em atletas nulíparas jogadoras da modalidade de futebol. No entanto no Grupo Controle, porém, a IU também esteve presente. Este dado levou à decisão de incluir um instrumento não validado, porém mais completo.

Em outro estudo a gravidade da IU ${ }^{21}$ foi estudada em um grupo de 47 mulheres fisicamente ativas, por meio do questionário ICIQ-SF, que avalia a frequência, gravidade e o impacto da IU. Foi observado perda de urina em 34 mulheres. A média do escore do questionário foi de 10,5 (entre 4 e 19 pontos). Esta pontuação revela uma IU classificada como moderada ( 6 a 12 pontos), sendo mais prevalente do que no presente estudo.

A IU mais prevalente foi a IUE já que, das 7 participantes, 2 relataram a perda ao exercitar-se e 2 em outras atividades de esforço. Corroborando com o estudo de Barreto et al. ${ }^{21}$ que observaram maiores ocorrências de perda de urina ocorrem durante a prática da atividade física, com prevalência de $72,3 \%$, seguido da tosse e do espirro com $35,2 \%$. Nossos achados confirmam que as mulheres fisicamente ativas apresentam com mais frequência a IUE.

Entre as limitações deste estudo, pontua-se que não foi possível atingir o tamanho amostral desejado por indisponibilidade para participar das voluntárias, - que impactou na ausência de significância nas 
análises estatísticas. Ressalta-se também a não utilização de um instrumento acurado para avaliar - nível de atividade física, de IU e de imagem corporal. Assim, não foi possível identificar se perda de urina ocorre devido à atividade exercida na academia ou pelos demais fatores de risco para IU, como a idade e os parâmetros dos treinamentos. Diante disso, recomenda-se a realização de novos estudos com amostras maiores e instrumentos validados para estimar com maior precisão a associação independente de variáveis que podem predizer a IU.

Entretanto, como a atividade física tem sido apontada como um importante fator de risco para IU, os presentes achados podem contribuir com a compreensão da IU em mulheres que praticam atividades físicas, despertando assim o interesse em pesquisas futuras e para sua prevenção.

\section{Conclusâo}

A prevalência de IU em mulheres praticantes de exercícios aeróbicos e musculação nas academias foi baixa (12\%), com severidade de moderada a grave e sem associação com idade, modalidade, tempo de prática, turno e frequência de exercícios. Recomenda-se cautela na interpretação destes achados devido à limitação do tamanho amostral.

\section{Contribuições dos autores}

Araújo NS participou da concepção, delineamento interpretação dos resultados. Silva LB e Santos WO participaram da redação do artigo científico. Rodrigues CNC participou da análise estatística dos dados da pesquisa, interpretação dos resultados. Nunes EFC participou da revisão e encaminhamento do artigo científico.

\section{Conflitos de interesses}

Nenhum conflito financeiro, legal ou político envolvendo terceiros (governo, empresas e fundações privadas, etc.) foi declarado para nenhum aspecto do trabalho submetido (incluindo mas não limitandose a subvenções e financiamentos, conselho consultivo, desenho de estudo, preparação de manuscrito, análise estatística, etc).

\section{Referências}

1. Fernandes S, Coutinho ECC, Duarte JC, Nelas PAB, Chaves CMCB, Amaral O. Qualidade de vida em mulheres com incontinência urinária. Revista de Enfermagem Referência. 2015; Série IV(5):93-99. doi: 10.12707/RIV14042

2. Dedicação AC, Haddad M, Saldanha MES, Driusso P. Comparação da qualidade de vida nos diferentes tipos de incontinência urinária feminina. Revista Brasileira de Fisioterapia. 2009;13(2):1 16-22. doi: 10.1590/\$1413$\underline{35552009005000014}$

3. dos Santos ES, Caetano AS, Tavares MCGCF, Lopes MHBM. Incontinência urinária entre estudantes de educação física. Rev Esc Enferm. 2009;43(2):307-12. doi: 10.1590/S0080$\underline{62342009000200008}$

4. Leroy LS, Lopes MHBM, Shimo AKK. A incontinência urinária em mulheres e os aspectos raciais. Texto Contexto Enfermagem. 2012;21(3):692-701. doi: 10.1590/S0104$\underline{07072012000300026}$

5. Silva DTG, Moraes NM. Estudo comparativo da força muscular do Assoalho Pélvico em mulheres sedentárias e mulheres que praticam atividade física [dissertação]. Belém: Centro de Ciências Biológicas e da Saúde da Universidade da Amazônia; 2006.

6. Caetano AS, Tavares MCGCF, Lopes MHBM. Incontinência Urinária e a Pratica de Atividade Física. Rev Bras Med Esporte. 2007;13(4):270-274. doi: 10.1590/S1517$\underline{86922007000400012}$

7. Barros JD, Lucena ACT, Anselmo CWSF. Incontinência Urinária de esforço em atletas do sexo feminino: Uma revisão da literatura. An Fac Med Univ Fed. Pernambuco. 2007;52(2):173-180.

8. Baracho E. Fisioterapia aplicada à Obstetrícia, Uroginecologia e aspectos de Mastologia. 4.ed. Rio de Janeiro: Guanabara Koogan; 2007.

9. de Araújo MP, de Oliveira E, Zucchi EVM, Trevisani VFM, Girão MJBC, Sartori MGF. Relação entre Incontinência Urinária em mulheres atletas corredoras de longa distância e distúrbio alimentar. Rev Assoc méd Bras. 2008;54(2):146-9. doi: $10.1590 /$ S0104-42302008000200018

10. Dockter M, Kolstad AM, Martin KA, Schiwal LJ. Prevalence of Urinary Incontinence: a comparative study of collegiate femaleathlets and non- athletic controls. Journal of women's health prysical therapy. 2007;31(1):12-17.

11. Hendriks EJ, Bernard ATM, Bie RA, Henrica CW. The psychometric properties of the PRAFAB-questionnaire: $A$ brief assessment questionnaire to evaluate severity of urinary incontinence in women. Neurourology and Urodynamics 2008;(27):379-387. 
12. Virtuoso JF, Menezes EC, Mazo GZ. Fatores de risco para incontinência urinária em mulheres idosas praticantes de exercícios físicos. Rev Bras Ginecol Obstet. 2015;37(2):82-6. doi: $10.1590 / \mathrm{SO} 100-720320140005040$

13. Korelo RIG, Kosiba CR, Grecco L, Matos RA. Influência do fortalecimento abdominal na função perineal, associado ou não à orientação de contração do assoalho pélvico, em nulíparas. Fisioter Mov. 2011 ;24(1):75-85. doi: 10.1590/ $\underline{\text { S0103-51502011000100009 }}$

14. de Almeida PP, Machado LRG. A prevalência de incontinência urinária em mulheres praticantes de jump. Fisioter Mov. 201 2;25(1):55-65. doi: 10.1590/S0103$\underline{51502012000100006}$

15. Moreno AL. Fisioterapia em uroginecologia. 2.ed. Barueri: Manole; 2009.

16. Goldstick $O$, Constantini N. Urinary incontinence in physically active women and female athetes. $\mathrm{Br} J$ Sport Med. 2014;48:296-298. doi:10.1136/bjsports-2012-091880

17. Botelho F, Silva $C$, Cruz F. Incontinência Urinária Feminina. Acta urológica, 2007;24(1):79-82.

18. de Sousa JG, Ferreira VR, de Oliveira RJ, Cestari CE. Avaliação da força muscular do assoalho pélvico em idosas com incontinência urinária. Fisioter Mov. 2011 ;24(1):39-46. doi: $10.1590 /$ S0103-51502011000100005

19. Melville JL, Katon W, Delaney K, Newton K. Urinary incontinence in US women a population-based study. Arch Intern Med. 2005; 165(14):537-42. doi: $10.1001 /$ archinte. 165.5.537

20. Filoni E. Avaliação da prevalência de sintomas de incontinência urinária em adolescentes mulheres praticantes de futebol e do impacto na qualidade de vida [tese]. Campinas: Faculdade de Ciências Médicas, Universidade Estadual de Campinas; 2013.

21. Barreto E, Filoni E, Fitz FF. Sintomas do trato urinário inferior em mulheres que praticam exercício físico regularmente. MTP\&Rehab Journal. 2014;(1 2):773-791. 